\title{
A Huge Thrombus-Containing Pulmonary Artery Aneurysm
}

\section{Mohamed Sanhoury*, Abdallah Almaghraby, Mohammed Ghazy, Yehia Saleh and Mostafa Nawar}

Department of Cardiology and Angiology, Faculty of Medicine, University of Alexandria, Egypt

\begin{abstract}
Pulmonary artery aneurysms are very rare entities that may be caused by several diseases. One of its causes is infection by Bilharziasis which is an endemic disease in Egypt. We report a case of a male patient presented by dyspnea and his chest $\mathrm{x}$-ray showed a huge pulmonary artery as well as an atelectatic lung lobe. Final diagnosis was done using echocardiography that showed a huge pulmonary artery aneurysm containing a very large organized thrombus.
\end{abstract}

Keywords: Bilhariziasis; Thrombosis; Pulmonary artery aneurysm

\section{Introduction}

Pulmonary artery aneurysms are very uncommon entities that may be caused by chest trauma, infections, and less commonly congenital or due to chronic pulmonary hypertension [1].

One of the infectious causes of pulmonary artery aneurysms is bilharzial or schistosomal infection, as Bilharziasis is endemic in our country Egypt, we are presenting a case of bilharzia-induced pulmonary artery aneurysm and thrombosis [2].

\section{Case Report}

A 55-year-old male patient presented to our facility complaining of dyspnea grade II and effort intolerance for several months. His medical history was unremarkable except for Bilharziasis since his childhood. On examination, he was hemodynamically stable and on auscultation accentuated P2 component of the second heart sound was heard with diastolic murmur over the pulmonary area indicating pulmonary regurgitation. His electrocardiogram showed sinus rhythm, right bundle branch block and right axis deviation. His chest X-ray

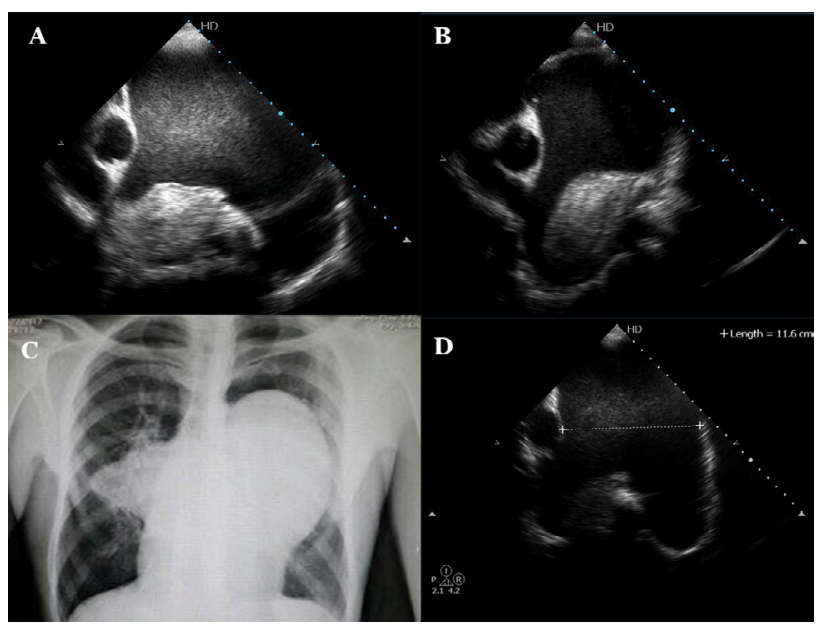

Figure 1: A: Transthoracic echocardiography, parasternal short axis at the level of the great vessels showing the large thrombus inside the main pulmonary artery. B: Transthoracic echocardiography, parasternal short axis at the level of the great vessels showing the extension of the thrombus into the main pulmonary artery branches. C: Chest X-ray, posterior anterior view showing the hugely dilated pulmonary artery as well as a large area of wedge pulmonary infarction caused by the distal embolization of the pulmonary artery thrombus. D: Transthoracic echocardiography, parasternal short axis at the level of the great vessels showing the degree of main pulmonary artery dilatation. showed a hugely dilated pulmonary artery as well as a large area of right lung wedge shaped infarction. Transthoracic echocardiography showed dilated right sided chambers with moderate tricuspid valve regurgitation showing a transvalvular peak systolic gradient of 57 $\mathrm{mmHg}$. The pulmonary artery was hugely dilated measuring $120 \mathrm{~mm}$ in its maximal diameter and showing a huge thrombus measuring 83 $\mathrm{mm} \times 47 \mathrm{~mm}$ in its maximal dimensions. Also, moderate pulmonary valve regurgitation was noted. The left ventricular systolic function was normal with a paradoxical septal motion coinciding with the right sided volume and pressure overload. Patient was put on anticoagulation in the form of Warfarin $4 \mathrm{mg}$ for 2 months and he is showing improvement of his symptoms on follow-up, but the thrombus is still present.

\section{Discussion}

Pulmonary artery aneurysms (PAA) are rare and infrequently diagnosed and the ideal treatment of PAA remains uncertain because there are no clear guidelines for the best therapeutic approach, due to limited experience because of the rarity of the disease.

In our case, the most probable etiology is the bilharzial infection where the schistosomal eggs are frequently embolized through the hepatic vein to the inferior vena cava then to the pulmonary arteries where they produce a type of chronic granulomatous inflammation that later on causes the pulmonary hypertension and the pulmonary artery aneurysm [2] (Figures 1A-1D).

Still the management of pulmonary artery thrombus is only through proper anticoagulation and follow-up with very limited role for surgery [2].

\section{Conclusion}

Though very rare, Bilharziasis should still be considered as one of the causes of pulmonary artery aneurysms and core-pulmonale especially in endemic areas.

*Corresponding author: Mohamed Sanhoury, Department of Cardiology and Angiology, Faculty of Medicine, University of Alexandria, Egypt, Tel: 201094844699; E-mail: drsanhory@yahoo.com

Received November 07, 2017; Accepted November 17, 2017; Published November 20, 2017

Citation: Sanhoury M, Almaghraby A, Ghazy M, Saleh Y, Nawar M (2017) A Huge Thrombus-Containing Pulmonary Artery Aneurysm. J Cardiovasc Dis Diagn 5: 298 doi: 10.4172/2329-9517.1000298

Copyright: (c) 2017 Sanhoury M, et al. This is an open-access article distributed under the terms of the Creative Commons Attribution License, which permits unrestricted use, distribution, and reproduction in any medium, provided the original author and source are credited. 
Citation: Sanhoury M, Almaghraby A, Ghazy M, Saleh Y, Nawar M (2017) A Huge Thrombus-Containing Pulmonary Artery Aneurysm. J Cardiovasc Dis Diagn 5: 298. doi: 10.4172/2329-9517.1000298

Page 2 of 2

\section{References}

1. Deterling RA, Clagett ОT (1947) Aneurysm of the pulmonary artery: Review of the literature and report of a case. Am Heart J 34: 471-499.
2. Morris W, Knauer CM (1997) Cardiopulmonary manifestations of schistosomiasis. Semin Respir Infect 12: 159-170. 